\title{
Cytoplasmic E-Cadherin Expression Is Associated With Higher Tumour Level of VEGFA, Lower Response Rate to Irinotecan-based Treatment and Poorer Prognosis in Patients With Metastatic Colorectal Cancer
}

\author{
RIYAD BENDARDAF ${ }^{1,2}$, FATEMEH SAHEB SHARIF-ASKARI ${ }^{3}$, \\ NARJES SAHEB SHARIF-ASKARI ${ }^{3}$, KARI SYRJÄNEN ${ }^{4}$ and SEPPO PYRHÖNEN ${ }^{5}$ \\ ${ }^{I}$ Oncology Unit, University Hospital Sharjah, Sharjah, United Arab Emirates; \\ ${ }^{2}$ Department of Clinical Sciences, College of Medicine, University of Sharjah, Sharjah, United Arab Emirates; \\ ${ }^{3}$ Sharjah Institute of Medical Research, College of Medicine, University of Sharjah, Sharjah, United Arab Emirates; \\ ${ }^{4}$ Department of Clinical Research, Biohit Oyj, Helsinki, Finland; \\ ${ }^{5}$ Department of Oncology and Radiotherapy, Turku University Hospital and University of Turku, Turku, Finland
}

\begin{abstract}
Background: The prognostic value of vascular endothelial growth factor-A (VEGFA) and epithelial cadherin ( $E$ cadherin) expression in patients with metastatic colorectal cancer ( $m C R C)$ is controversial. Materials and Methods: In this prospective study, patients diagnosed with $m C R C$ between August 1, 1998, and August 30, 2003, at the Turku University Hospital, Finland were included. Expression of E-cadherin (membranous and cytoplasmic pattern) and VEGFA in tumour samples was assessed by immunohistochemistry. Tumours were classified as E-cadherin expressers if they demonstrated moderate or strong cytoplasmic or membranous staining, while those positive for VEGFA expression showed a moderate or strong cytoplasmic staining. Of particular interest was the association between membranous or cytoplasmic expression of E-cadherin and VEGFA. The value of strong VEGF-A staining and membranous or cytoplasmic expression of E-cadherin as a predictor of disease outcome over a 6-year period was another point of interest in this study. Results: Of the 67 patients with $m C R C, 43$ (64\%) had tumours positive for cytoplasmic $E$ cadherin, while in 24 cases (36\%), E-cadherin expression was membranous. Strong VEGFA staining was present in half of the cases ( $n=36,54 \%$ of all $67 \mathrm{mCRC}$ cases). VEGFA expression
\end{abstract}

This article is freely accessible online.

Correspondence to: Fatemeh Saheb Sharif-Askari, Sharjah Institute of Medical Research, College of Medicine, University of Sharjah, Sharjah, United Arab Emirates. Tel: +971 501829166, e-mail: fsharifaskari@sharjah.ac.ae

Key Words: Metastatic colorectal cancer, E-cadherin, VEGFA, irinotecan regimen. was significantly correlated with cytoplasmic E-cadherin expression in that 28/36 cases of VEGFA-positive tumours were also positive for cytoplasmic E-cadherin $(p=0.012)$. In addition, among the patients with intense VEGFA expression ( $n=36)$, those who had positive cytoplasmic E-cadherin in their tumours had a lower response-rate to first-line therapy with irinotecan, fluorouracil and leucovorin regimen: 5 out of 36 (14\%) were chemosensitive. This is in contrast to the patients with VEGFApositive tumours and membranous E-cadherin (8/36, 22\% chemosensitive $(p=0.004)$. The former group also had more ominous prognosis $(p<0.001)$. Conclusion: Reduced membranous expression of E-cadherin and increased cytoplasmic E-cadherin expression predict poor survival in $\mathrm{mCRC}$.

Colorectal cancer (CRC) is the third most common cancer (1), and is one of the leading causes of mortality and morbidity worldwide (2). Although comprehensive treatment is available, including curative resection and chemotherapy, the prognosis of CRC is still far from optimal. Several common clinicopathological factors, such as tumour grade, perineural and lymphovascular invasion, microsatellite instability status, Kirsten rat sarcoma viral oncogene (KRAS), neuroblastoma RAS viral oncogene homolog (NRAS), and v-raf murine sarcoma viral oncogene homolog $\mathrm{B}(B R A F)$ mutations, circumferential resection margin, and carcino-embryonic antigen (CEA) have been extensively tested as prognostic predictors $(3,4)$. Some of these factors can be useful in the selection of patients for treatment but most of them are of limited value in predicting the individual response to treatment or overall outcomes. Therefore, the search still continues for new prognostic markers to guide therapy and improve the survival rates of patients with CRC. The idea of finding a single marker suitable for all purposes is obsolete, and the goal should be to move towards a personalized 
therapy of patients, based on prognostic markers that should accurately predict the response to therapy.

E-Cadherin, a metastatic suppressor gene, is a calciumdependent adhesive protein responsible for epithelial cell cohesion and also participating in cellular signalling, albeit the nature of these intracellular signalling pathways has not been fully elucidated. However, increasing evidence assigns an important role to activation of the WNT signalling pathway and its major mediator cadherin-associated protein, $\beta$-catenin, in normal and pathogenic angiogenesis (5). Given that adenomatous polyposis coli (APC) is a tumour suppresser in human colon cancer and its mutation enhances $\beta$-catenin gene expression $(6,7)$, there is increasing evidence to suggest an involvement of the cadherin/catenin complex disruption in a variety of human cancer types (8-10). It was reported that increased cytoplasmic staining of cadherincatenin complex is an independent predictor of poor survival, whereas expression at the cell membrane is not $(11,12)$.

Overexpression of $\beta$-catenin also produces an increase in cellular vascular endothelial growth factor A (VEGFA) protein (13), a key mediator of angiogenesis (14). VEGFA is regulated by few interacting cellular pathways such as transforming growth factor beta (TGF $\beta$ ) signalling, WNT/ $\beta$-catenin, and VEGF pathways that are necessary for embryonic vasculogenesis and angiogenesis (14). In particular, mutation in canonical WNT/ $\beta$-catenin signalling components including $A P C$, axin, and $\beta$-catenin have been reported in gastrointestinal malignancies (15), and results in hyperactivation of the WNT pathway, ultimately increasing proliferation of malignant cells and angiogenesis $(13,14)$. Experimental studies have demonstrated that $\beta$-catenin directly induces VEGFA expression when normal colon epithelial cells were transfected with mutant form of $\beta$-catenin gene (13). Therefore, $\beta$-catenin is known to have dual effects, regulating cell proliferation when in the nucleus and cell adhesion when at the cell surface, both key mediators of angiogenesis (14). Although the individual prognostic value of E-cadherin and VEGFA in patients with CRC has been studied $(16,17)$, the combined prognostic value of VEGFA and E-cadherin expression in patients with mCRC has not been evaluated, to our knowledge. In the present study, we analysed the prognostic role of E-cadherin and VEGFA in primary tumours resected from patients with CRC, with special reference to evaluating the association between their immunohistochemical expression pattern and patient outcomes.

\section{Materials and Methods}

Study population. The material of this study consisted of CRC specimens from 67 patients who had undergone resection at the Turku University Hospital between 1998 and 2003. Of the patients, 43 were men and 24 were women, aged 24-80 years (mean age $=57.6$ years; Table I). No patients had received irradiation or chemotherapy prior to resection. Forty-two patients were diagnosed as having metastatic disease at time of diagnosis (stage IV), while
Table I. Baseline characteristics of the study cohort ( $n=67)$.

\begin{tabular}{|c|c|}
\hline Characteristic & Value \\
\hline \multicolumn{2}{|l|}{ Age, years } \\
\hline Mean \pm SD (range) & $57.6 \pm 11.5(24-80)$ \\
\hline \multicolumn{2}{|l|}{ Gender, n (\%) } \\
\hline Female & $24(36)$ \\
\hline Male & $43(64)$ \\
\hline \multicolumn{2}{|c|}{ Location of the primary tumour, $\mathrm{n}(\%)$} \\
\hline Right colon & $15(22.4)$ \\
\hline Transverse colon & $7(10.4)$ \\
\hline Left colon & $31(46.3)$ \\
\hline Rectum & $14(20.9)$ \\
\hline \multicolumn{2}{|l|}{ TNM classification, $\mathrm{n}(\%)$} \\
\hline \multicolumn{2}{|l|}{ Tumour status } \\
\hline $\mathrm{T} 1$ & 0 \\
\hline $\mathrm{T} 2$ & $5(7.5)$ \\
\hline $\mathrm{T} 3$ & $48(71.6)$ \\
\hline $\mathrm{T} 4$ & $14(20.9)$ \\
\hline \multicolumn{2}{|l|}{ Nodal status } \\
\hline No & $18(26.9)$ \\
\hline $\mathrm{N} 1$ & $35(52.2)$ \\
\hline Unknown & $14(20.9)$ \\
\hline \multicolumn{2}{|l|}{$\begin{array}{l}\text { Location of metastases upon } \\
\text { starting chemotherapy, } \mathrm{n}(\%)\end{array}$} \\
\hline Local & $4(6)$ \\
\hline Liver & $28(41.8)$ \\
\hline Lung & $1(1.5)$ \\
\hline Multiple sites & $34(50.7)$ \\
\hline \multicolumn{2}{|l|}{ Histological grade, $\mathrm{n}(\%)$} \\
\hline I & $10(14.9)$ \\
\hline II & $45(67.2)$ \\
\hline III & $12(17.9)$ \\
\hline \multicolumn{2}{|l|}{ Stage, n (\%) } \\
\hline II & $11(16.4)$ \\
\hline III & $14(20.9)$ \\
\hline IV & $42(62.7)$ \\
\hline \multicolumn{2}{|c|}{ Tumour immunohistochemistry, n (\%) } \\
\hline Strong VEGFA & $36(53.7)$ \\
\hline Cytoplasmic E-cadherin & $43(64.1)$ \\
\hline \multicolumn{2}{|c|}{ Disease-specific survival (months) } \\
\hline Mean \pm SD & $34.4 \pm 26.6$ \\
\hline \multicolumn{2}{|c|}{ Disease-specific outcome, $\mathrm{n}(\%)^{*}$} \\
\hline Alive & $3(4.5)$ \\
\hline Died of disease & $64(95.5)$ \\
\hline
\end{tabular}

SD: Standard deviation, VEGFA: vascular endothelial growth factor A.*By end of study.

Table II. Association between vascular endothelial growth factor A (VEGFA) staining and E-cadherin expression in patients with metastatic colorectal cancer

\begin{tabular}{lcccc}
\hline & \multicolumn{3}{c}{ E-Cadherin, $\mathrm{n}(\%)$} \\
\cline { 3 - 4 } VEGFA & Total, $\mathrm{n}$ & Cytoplasmic & Membranous & \\
\hline Strong & 36 & $28(78)$ & $8(22)$ & 0.012 \\
Weak & 31 & $15(48)$ & $16(52)$ & \\
\hline
\end{tabular}




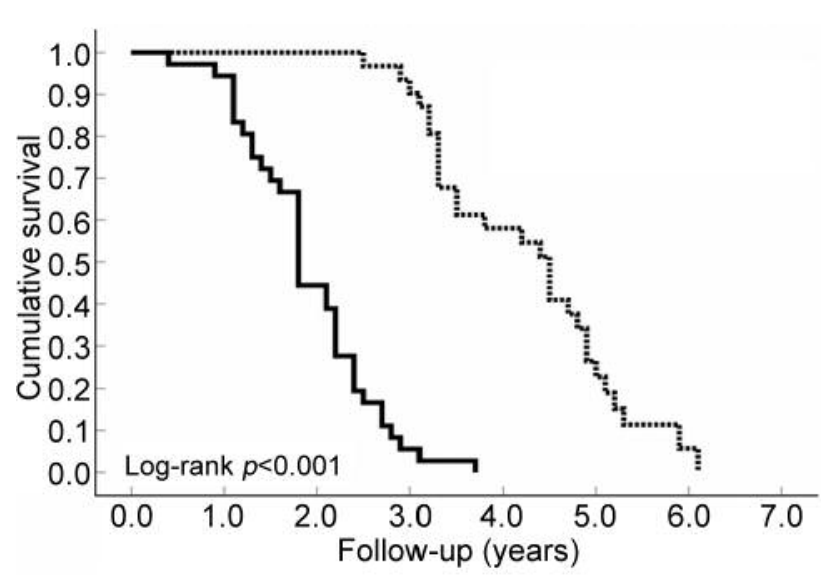

.* Weak VEGFA staining

$\sim$ Strong VEGFA staining

Figure 1. Overall survival of patients with metastatic colorectal cancer according to vascular endothelial growth factor A (VEGFA) expression.

the remaining 25 patients with stage II $(n=11)$ and III $(n=14)$ developed metastasis later during the follow-up period. Written informed consent to participate in the study was obtained from each patient before surgery. The study was approved by the National Authority for Medico-Legal Affairs Committee, Finland and was conducted in accordance with the declaration of Helsinki.

Immunohistochemical staining. A formalin-fixed, paraffin-embedded primary tumour was obtained from each patient. Sections were cut serially at $5 \mu \mathrm{m}$ for routine haematoxylin and eosin (HE) staining and for immunohistochemical analysis. An experienced pathologist confirmed all histological diagnoses. Evaluation and scoring of the immunohistochemical slides was carried out without knowledge of the clinical data.

Evaluation of E-cadherin expression. Expression of E-cadherin was studied using monoclonal mouse antibody to E-cadherin (Clone HECD-1, sub-class IgG1; Zymed Laboratories, San Francisco, CA, USA) at a dilution of 1:300 in phosphate-buffered saline containing $1 \%$ bovine serum albumin (1:50). Two independent observers assessed the expression of E-cadherin. The slides were first screened for an overview of the general staining pattern. Cytoplasmic expression of E-cadherin was recorded when staining was absent from the cancer cell membrane and preserved in cancer cell cytoplasm and nucleus.

Evaluation of VEGFA expression. For studying VEGFA expression, purified anti-human VEGFA (121, 165, and 189 isoforms) (clone VG-1; Biosite, San Diego, CA, USA) (1:150) was used. The expression of VEGFA in the tumour tissue was assessed according to the expression in the total tumour area. Only cytoplasmic staining was observed, and graded into four categories: negative, no detectable staining; weak but detectable staining; moderate, clearly positive staining; and strong staining, intense throughout the tumour.

Evaluation of patient response to the first-line therapy with irinotecan, fluorouracil and leucovorin IFL. All patient received IFL

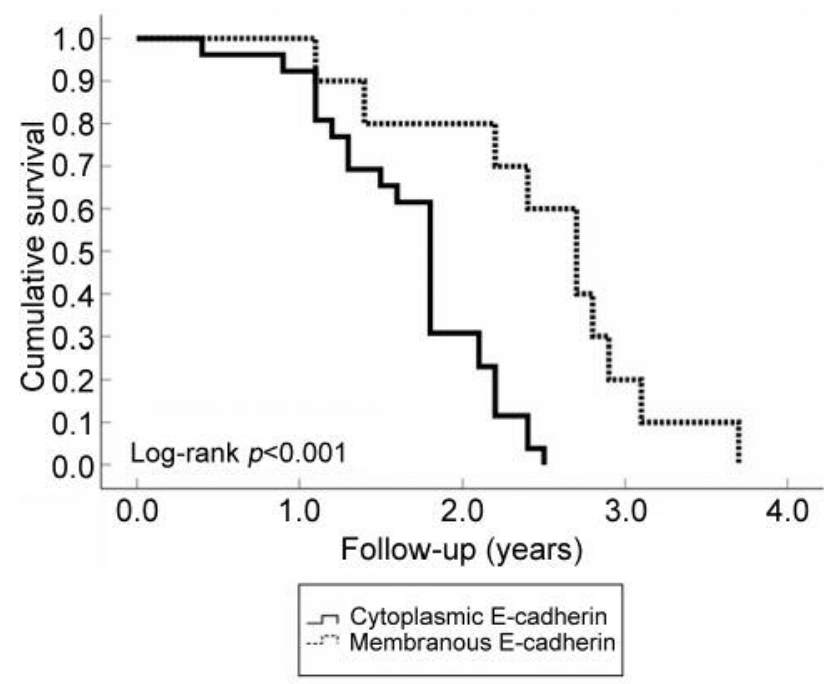

Figure 2. Overall survival of patients with metastatic colorectal cancer according to E-cadherin in patients with tumours with strong vascular endothelial growth factor A (VEGFA) expression.

regiment once they developed metastasis. The IFL treatment was a combination of irinotecan $\left(180-210 \mathrm{mg} \mathrm{m}^{-2}\right.$, administered as a $60-$ to 90 -min intravenous infusion) and 5-fluorouracil (500 $\mathrm{mg} \mathrm{m}^{-2}$, i.v. bolus), modulated by leucovorin $\left(60 \mathrm{mg} \mathrm{m}^{-2}\right.$, i.v. bolus $)$. The $5-$ flurouracil/leucovorin administration was repeated on the following day. This treatment combination was repeated every 2 weeks until disease progression or occurrence of unacceptable toxicity. Tumour response was assessed every 8 weeks by an independent reviewer according to WHO criteria $(18,19)$.

Statistical analysis. All analyses were performed with SPSS 21.0 (IBM, Armonk, NY, USA), and Sample Power 3.0 (SPSS). Categorical variables are presented herein as percentages, and continuous variable (age) are presented as means $( \pm \mathrm{SD})$. The age variable was checked for normal distribution using the Kolmogorov-Smirnov test. The expression of E-cadherin was used as a dichotomous variable, where tumours with nuclear, heterogeneous or cytoplasmic expression of Ecadherin were one category (cytoplasmic E-cadherin), and all those with well-localized membranous expression of E-cadherin were grouped into the second category (membranous E-cadherin). The expression of VEGFA was also classified as a binary variable, whereby tumours with negative or weak staining were considered as exhibiting weak VEGFA expression, and those with moderate or strong staining were considered as having strong VEGFA expression. Comparisons of categorical variables were performed using the chi-square test or Fisher's exact test. Survival curves were computed according to Kaplan and Meier; the log-rank test was used to examine the difference between the curves. A $p$-value of less than 0.05 was accepted as statistically significant for all tests.

\section{Results}

Patterns of E-cadherin expression. Of the 67 tumour samples studied, 43 cases $(64 \%)$ showed cytoplasmic E-cadherin and 24 cases $(36 \%)$ were positive for membranous E-cadherin. 
Correlation between VEGFA and E-cadherin expression. VEGFA was significantly correlated with cytoplasmic Ecadherin expression, with 28 out of 36 VEGFA-positive tumours showing cytoplasmic E-cadherin expression and only 8/36 showing membranous E-cadherin expression (Table II) $(p=0.012)$.

Correlation between VEGFA and E-cadherin expression in relation with response to first line IFL and overall survival $(O S)$. During the 6-year follow-up, 31 out of 67 patients responded to IFL regimen: 18 out of 31 were patients with weak VEGFA tumour staining, and 13 were patients with strong VEGFA tumour staining. Patients with strong VEGFA tumour staining had a lower OS rate following the first-line IFL regimen (all 36 had died, as compared to the patients with weak VEGFA tumour staining (28; 90\% died) $(p<0.001)$ (Figure 1). Among the patients with strong VEGFA tumour staining $(n=36)$, those who had cytoplasmic E-cadherin-positive tumours had a lower response rate to the first-line IFL regimen (5/36, $14 \%$ were chemosensitive) as compared to those with VEGFA tumours with membranous E-cadherin expression $(8 / 36,22 \% ; p=0.004)$. The former also had much worse prognosis $(p<0.001$; Figure 2$)$.

\section{Discussion}

The present study provides new insights into the role of cytoplasmic E-cadherin as a marker of treatment response in mCRC. In the present series of 67 patients with $\mathrm{mCRC}$, there was a significant association between cytoplasmic E-cadherin and VEGFA expression. Compared to the patients with tumours positive for membranous E-cadherin, the patients with cytoplasmic E-cadherin expression had a higher level of VEGFA co-expression, a lower response rate to the first-line IFL, and a poorer prognosis during the 6-year follow-up.

In this study, the cytoplasmic localization of E-cadherin was co-expressed with VEGFA, suggesting that loss of membranous E-cadherin promotes tumour angiogenesis. Similarly, Ceteci et al. who studied the role of E-cadherin in early development of lung cancer revealed that disruption of E-cadherin up-regulates angiogenesis target genes VEGFA and VEGFC, leading to massive intratumoural vessels formation in the early phase of tumour induction in RAFdriven murine lung cancer (20). In their study, $\beta$-catenin was identified as an effector of E-cadherin disruption. The simple fact is that E-cadherin traps $\beta$-catenin at the cytoplasm and loss of this interaction enables nuclear translocation of $\beta$-catenin and angiogenesis through WNT pathway activation (21). Moreover, in their study induction of VEGFA, not VEGFC was $\beta$-catenin-dependent (20). Consistent with data reported by Zhang et al., who demonstrated that VEGFA promotor activity could be stimulated by oncogenic $\beta$-catenin in HeLa cells (22), and by Easwaran et al., who described VEGFA as a direct target gene of $\beta$-catenin in human colorectal cancer cell lines (13). The reasonable explanation for these findings could be a signalling cascade connecting E-cadherin disruption via $\beta$-catenin with angiogenesis target gene VEGFA that can be considered as a hallmark of cancer progression.

Another noteworthy finding in our study is that among the patients with strong VEGFA expression, positive cytoplasmic E-cadherin expression was a predictor of chemotherapyrefractory status and more ominous prognosis, whereas membranous E-cadherin expression had no such associations. Consistent with other studies on different human cancer types, loss of membranous expression of E-cadherin was significantly associated with a lower response to chemotherapy and lower OS rate (23-25). There are several possible reasons associated with the chemotherapy-refractory status, one is loss of membranous E-cadherin or cell-cell junction efficiency in cancer cells resulting in derailed endocytosis of surface receptors and reduced effectiveness of chemotherapy drugs (26). Another involves disruption of E-cadherin activation of $\beta$-catenin signalling (20), which can profoundly affect the genetic composition of the tumour cells (5) and influence the response to chemotherapy. As an example, recent observations point to the fact that when the tumour-suppressor gene $p 53$ is overexpressed as a defence mechanism against tumour growth, the oncogenic capabilities of $\beta$-catenin are reduced. There is a selective pressure for the tumours to inactivate p53 in order to sustain activation of the $\beta$-catenin signalling $(27,28)$. Therefore, this could be responsible for the lower response rate to irinotecan-based regimen because it has been demonstrated that irinotecan-induced apoptosis was inhibited by reduced p53 expression (29).

In conclusion, E-cadherin and VEGFA combination might be used as a prognostic marker in mCRC. Reduction in membranous expression of E-cadherin and increased cytoplasmic E-cadherin expression seem to predict poor survival in mCRC.

\section{Conflicts of Interest}

There are no conflicts of interest regarding this study.

\section{Authors' Contributions}

Study design and experimental work: R.B.; Analysis and article writing: F.S.S. and N.S.S.; analysis and article revision: K.S. and S.P.

\section{References}

1 Siegel RL, Miller KD and Jemal A: Cancer statistics, 2016. CA Cancer J Clin 66(1):7-30, 2016. PMID: 26742998. DOI: 10.3322/caac. 21332

2 Bhandari A, Woodhouse M and Gupta S: Colorectal cancer is a leading cause of cancer incidence and mortality among adults 
younger than 50 years in the USA: A SEER-based analysis with comparison to other young-onset cancers. J Investig Med 65(2): 311-315, 2017. PMID: 27864324. DOI: 10.1136/jim-2016-000229

3 Edge SB and Compton CC: The American Joint Committee on Cancer: The 7th edition of the AJCC cancer staging manual and the future of TNM. Ann Surg Oncol 17(6): 1471-1474, 2010. PMID: 20180029. DOI: 10.1245/s10434-010-0985-4

4 Fichera A: Less is more in colorectal cancer post-treatment surveillance. JAMA Surg 153(10): 877, 2018. PMID: 29971431. DOI: 10.1001 /jamasurg.2018.2066

5 Goodwin $\mathrm{AM}$ and d'Amore PA: WNT signaling in the vasculature. Angiogenesis 5: 1-9, 2002. PMID: 12549854.

6 Morin PJ, Sparks AB, Korinek V, Barker N, Clevers H, Vogelstein B, and Kinzler KW: Activation of $\beta$-catenin-TCF signaling in colon cancer by mutations in $\beta$-catenin or $A P C$. Science 275(5307): 1787-1790, 1997. PMID: 9065402.

7 Chan TA, Wang Z, Dang LH, Vogelstein B, and Kinzler KW: Targeted inactivation of CTNNB1 reveals unexpected effects of beta-catenin mutation. Proc Natl Acad Sci USA 99(12): 82658270, 2002. PMID: 12060769. DOI: 10.1073/pnas.082240999.

8 Salon C, Lantuejoul S, Eymin B, Gazzeri S, Brambilla C, and Brambilla E: The E-cadherin- $\beta$-catenin complex and its implication in lung cancer progression and prognosis. Future Oncol 1(5): 649-660, 2005. PMID: 16556042. DOI: 10.2217/ 14796694.1.5.649

9 Jawhari A, Farthing M, and Pignatelli M: The importance of the E-cadherin-catenin complex in the maintenance of intestinal epithelial homoeostasis: More than intercellular glue? Gut 41(5): 581-584, 1997. PMID: 9414960.

10 Wijnhoven BPL, Dinjens WNM and Pignatelli M: E-Cadherincatenin cell-cell adhesion complex and human cancer. Br J Surg 87(8): 992-1005, 2000. PMID: 10931041. DOI: 10.1046/j.13652168.2000.01513.x

11 Ramesh S, Nash J and McCulloch PG: Reduction in membranous expression of $\beta$-catenin and increased cytoplasmic E-cadherin expression predict poor survival in gastric cancer. $\mathrm{Br}$ J Cancer 81(8): 1392-1397, 1999. PMID: 10604738. DOI: $10.1038 /$ sj.bjc. 6693437

12 Kim SA, Inamura K, Yamauchi M, Nishihara R, Mima K, Sukawa Y, Li T, Yasunari M, Morikawa T and Fitzgerald KC: Loss of CDH1 (E-cadherin) expression is associated with infiltrative tumour growth and lymph node metastasis. Br J Cancer 114(2): 199-206, 2016. PMID: 10604738. DOI: 10.1038/ sj.bjc. 6693437

13 Easwaran V, Lee SH, Inge L, Guo L, Goldbeck C, Garrett E, Wiesmann M, Garcia PD, Fuller JH and Chan V: $\beta$-Catenin regulates vascular endothelial growth factor expression in colon cancer. Cancer Res 63 (12): 3145-3153, 2003. PMID: 12810642.

14 Clifford RL, Deacon K and Knox AJ: Novel regulation of vascular endothelial growth factor-A (VEGF-A) by transforming growth factor (beta) 1: Requirement for SMADs, beta-catenin, and GSK3(beta). J Biol Chem 283(51): 35337-35353, 2008. PMID: 18952601. DOI: 10.1074/jbc.M803342200

15 Flanagan DJ, Austin CR, Vincan E and Phesse TJ: WNT signalling in gastrointestinal epithelial stem cells. Genes 9(4): 178, 2018. PMID: 29570681. DOI: 10.3390/genes9040178

16 Bendardaf R, Elzagheid A, Lamlum H, Ristamäki R, Collan Y and Pyrhönen S: E-Cadherin, CD44s and CD44v6 correlate with tumour differentiation in colorectal cancer. Oncol Rep 13: 831835, 2005. PMID: 15809746.
17 Bendardaf R, Buhmeida A, Hilska M, Laato M, Syrjänen S, Syrjänen K, Collan Y and Pyrhönen S: VEGF-1 expression in colorectal cancer is associated with disease localization, stage, and long-term disease-specific survival. Anticancer Res 28: 3865-3870, 2008. PMID: 19192642.

18 Miller AB, Hoogstraten B, Staquet M and Winkler A: Reporting results of cancer treatment. Cancer 47(1): 207-214, 1981. PMID: 7459811.

19 Therasse P, Arbuck SG, Eisenhauer EA, Wanders J, Kaplan RS, Rubinstein L, Verweij J, Van Glabbeke M, van Oosterom AT and Christian MC: New guidelines to evaluate the response to treatment in solid tumors. J Natl Cancer Inst 92(3): 205-216, 2000. PMID: 10655437.

20 Ceteci F, Ceteci S, Karreman C, Kramer BW, Asan E, Götz R and Rapp UR: Disruption of tumor cell adhesion promotes angiogenic switch and progression to micrometastasis in RAFdriven murine lung cancer. Cancer Cell 12(2): 145-59, 2007. PMID: 17692806. DOI: 10.1016/j.ccr.2007.06.014

21 Nelson WJ and Nusse R: Convergence of WNT, ß-catenin, and cadherin pathways. Science 303(5663): 1483-7, 2004. PMID: 115001769. DOI: $10.1126 /$ science.1094291

22 Zhang X, Gaspard JP and Chung DC: Regulation of vascular endothelial growth factor by the WNT and K-RAS pathways in colonic neoplasia. Cancer Res 61(16): 6050-6054, 2001. PMID: 11507052 .

23 Koensgen D, Freitag C, Klaman I, Dahl E, Mustea A, Chekerov R, Braicu I, Lichtenegger W and Sehouli J: Expression and localization of E-cadherin in epithelial ovarian cancer. Anticancer Res 30(7): 2525-30, 2010. PMID: 20682978.

24 Li Z, Yin S, Zhang L, Liu W and Chen B: Prognostic value of reduced E-cadherin expression in breast cancer: A meta-analysis. Oncotarget 8(10): 16445-16455, 2017. PMID: 28147315. DOI: 10.18632 /oncotarget.14860

25 Chen J, Zhao J, Ma R, Lin H, Liang X and Cai X: Prognostic significance of E-cadherin expression in hepatocellular carcinoma: A meta-analysis. PLoS ONE 9(8): e103952, 2014. PMID: 25093414. DOI: 10.1371/journal.pone.0103952

26 Mosesson Y, Mills GB and Yarden Y: Derailed endocytosis: An emerging feature of cancer. Nat Rev Cancer 8(11): 835, 2008. PMID: 18948996. DOI: 10.1038/nrc2521

27 Levina E, Oren M and Ben-Ze'ev A: Downregulation of $\beta$ catenin by $\mathrm{p} 53$ involves changes in the rate of $\beta$-catenin phosphorylation and axin dynamics. Oncogene 23(25): 44444453, 2004. PMID: 15064706. DOI: 10.1038/sj.onc.1207587

28 Riascos-Bernal DF, Chinnasamy P, Cao LL, Dunaway CM, Valenta T, Basler $\mathrm{K}$ and Sibinga NES: $\beta$-Catenin C-terminal signals suppress $\mathrm{p} 53$ and are essential for artery formation. Nat Commun 7: 12389, 2016. PMID: 27499244. DOI: 10.1038/ ncomms 12389

29 Takeba Y, Sekine S, Kumai T, Matsumoto N, Nakaya S, Tsuzuki Y, Yanagida Y, Nakano H, Asakura T and Ohtsubo T: Irinotecaninduced apoptosis is inhibited by increased P-glycoprotein expression and decreased p53 in human hepatocellular carcinoma cells. Biol Pharm Bull 30: 1400-1406, 2007. PMID: 17666793.
Received January 22, 2019

Revised March 9, 2019

Accepted March 13, 2019 